\title{
Stress ratio effect on tension-tension fatigue behavior of angle-ply GFRP laminates
}

\author{
A. Vahid Movahedi-Rad, Thomas Keller, Anastasios P. Vassilopoulos ${ }^{1}$ \\ Composite Construction Laboratory (CCLab), \\ Ecole Polytechnique Fédérale de Lausanne (EPFL), \\ Station 16, Bâtiment BP, CH-1015 Lausanne, Switzerland
}

\begin{abstract}
The effect of the stress ratio $\left(R=\sigma_{\min } / \sigma_{\max }\right)$ on the fatigue behavior of $( \pm 45)_{2}$ angle-ply glass/epoxy composite laminates was investigated by comparing their mechanical, thermal, and optical properties under the stress ratio of 0.5 with previous fatigue results obtained under the stress ratio of 0.1 . When the stress ratio was increased from 0.1 to 0.5 , the fatigue life was enhanced at the same $\sigma_{\max }$ and the slope of S-N curve decreased, exhibiting more scattered responses. In addition, as the stress ratio increased, the fatigue damage was distributed more uniformly with a lower self-generated temperature at the same $\sigma_{\max }$. At $R=0.5$, fiber realignment, due to cyclic creep, increased the fatigue stiffness, thus compensating the decreasing effect of fatigue damage. At high stress levels, the stiffening effect dominated the stiffness evolution, resulting in greater fatigue stiffness with an increasing number of fatigue cycles; however, at low stress levels the degrading effect due to fatigue damage was prevalent. The stiffening effect led to smaller hysteresis loop areas at the beginning of the fatigue experiments, which subsequently stabilized for the greater part of the specimen fatigue life, followed by a slight increase before failure. At $R=0.1$, the fatigue stiffness decreased further and the hysteresis loop area became larger at all stress levels at the same $\sigma_{\max }$ since the stiffening effect was less and fatigue damage more severe.
\end{abstract}

\section{Keywords:}

Fatigue; composites; stress ratio; fatigue stiffness; hysteresis loop area

\footnotetext{
${ }^{1}$ Corresponding author: e-mail: anastasios.vassilopoulos@epfl.ch,Tel: +41216936393; Fax: +41216936240.
} 


\section{Introduction}

It is widely accepted that fatigue is one of the most common failure types, especially in loadtransferring parts of structures operating in the open air. The main composite components of engineering structures are manufactured from materials whose performance may be matrix-dominated. Typical examples are shear webs and the aerodynamic parts of wind turbine rotor blades that consist mainly of biaxial composite laminates The structural integrity of such components is vital for design purposes and therefore researchers have devoted great efforts to understand their fatigue behavior $[1,2,3,4,5]$. Fiber-reinforced polymer (FRP) composites operating in open-air applications are subjected to different irregular loading profiles of a stochastic nature, comprising variable amplitude, and interrupted loading spectra $[6,7,8]$. Nevertheless, since it is impossible to experimentally investigate the fatigue behavior of each material of interest under all possible loading conditions, standard continuous fatigue experiments are performed in laboratories under different stress ratios in order to characterize the fatigue performance of each material for structural design purposes [3].

During fatigue loading, the mechanical properties of specimens are degraded as a result of the activation of various damage mechanisms, including fiber breakage and matrix cracking, debonding, transverse-ply cracking, and delamination $[9,10]$. Therefore, several parameters, such as stress level, loading frequency, stress ratio $\left(R=\sigma_{\min } / \sigma_{\max }\right)$, and material type, must be considered in order to appropriately design a fatigue-testing program. It has been well documented that stress level significantly affects the fatigue life of composites [10,11,12,13,14,15]. Moreover, it was recently shown that the different stress levels in constant amplitude fatigue experiments could cause different damage distributions in the specimen volume, and result in different stiffness degradation levels, hysteresis loop areas, and final fracture surfaces [10]. Any frequency increase has been shown to improve the fatigue life of several material systems as long as this increased frequency did not produce significant self-generated temperature increases that softened the matrix and decreased the fatigue life $[16,17,18,19,20]$.

The stress ratio also exerts a considerable effect on fatigue behavior, which is mainly attributed to the interaction between the time- and cyclic-dependent mechanical properties, since laminated composites creep under fatigue mean stress even at room temperature [10,26,27,28,29,30,31]. Under $R=1$, a specimen is loaded in pure creep. As the stress ratio decreases (in tensile-tensile fatigue), the stress amplitude increases, which leads to fewer cyclic creep effects and greater fatigue damage, and finally under $R=-1$ the specimen undergoes only fatigue damage [35,36]. In continuous fatigue, the interaction 
between the cyclic creep and fatigue damage has mainly been studied by monitoring the evolution of the fatigue hysteresis loops. In this case, the shift of the fatigue hysteresis loops represents the cyclic creep effects and the degradation of fatigue stiffness, which is the slope of the hysteresis loops, represents the damage effect [26,32,33,34]. The interaction degree between the cyclic creep and fatigue damage and dominance of one over the other also depends strongly on the material type. In matrix-dominated composites, the viscoelastic deformation is very significant, which leads to greater cyclic creep effects than in fiber-dominated composites.

To simulate the stress ratio effect on fatigue life, theoretical models, the so-called constant life diagrams (CLDs), were established [3,21,22,23,24,25]. A comparison of the simulation capabilities of the most commonly used models for composite materials was presented in [23].

Previous investigations of angle-ply laminated composites under tension-tension fatigue are limited to stress ratios of 0.1 , or 0 , while little information regarding the evolution of fatigue-related mechanical properties (e.g. fatigue strength and stiffness) and response (e.g. dissipated energy, cyclic creep strain) is available at other stress ratios $[2,10,13,15,30,37,38,39]$. Moreover, the majority of the works dealing with fatigue under higher stress ratios mainly focused on the development of relevant fatigue life prediction methodologies, and not on the thorough investigation of the effects of the $R$-ratio on the damage accumulation and cyclic- and time-dependent mechanical properties $[1,12,21,22,27,40]$.

The aim of this work is to investigate the stress ratio effect on the fatigue behavior of matrixdominated laminated composites in which the stress ratio effect on fatigue behavior is considerable. To achieve this objective, a dedicated experimental program was conducted to investigate the behavior of $\pm 45^{\circ}$ angle-ply GFRP composite laminates at a stress level of $R=0.5$. The variation of the fatigue stiffness, hysteresis loop areas, and cyclic creep was monitored by measuring the load and displacement in each fatigue cycle. In addition, the evolution of the fatigue damage and specimen surface temperature was recorded during the cyclic loading. The results were then compared to previous fatigue results obtained under $R=0.1$ [10] and the effect of the $R$-ratio has been thoroughly discussed.

\section{Experimental procedure}

\subsection{Material and specimen preparation}

Unidirectional E-glass fiber fabrics (EC 9-68) with an area density of $425 \mathrm{gr} / \mathrm{m}^{2}$ and layer thickness of $0.45 \mathrm{~mm}$ were used. These fabrics comprise a finish-bonding agent, which provides better adhesion to 
the matrix. The low viscosity resin, Biresin ${ }^{\circledR}$ CR83, mixed with the hardener Sika CH83-2 in a ratio of $3: 1$, was used for the impregnation of the fabrics. The fabrication was carried out on a plastic substrate, which was coated with a release agent to prevent the resin from bonding to the surface after fabrication. Laminates of $500 \times 500 \times 2.25 \mathrm{~mm}^{3}$ and stacking sequence of $[ \pm 45]_{2 \mathrm{~s}}$ were fabricated by vacuum-assisted hand lay-up, using a vacuum pump with pressure of 0.9 bar. Each laminate was placed in vacuum for 24 hours under laboratory conditions $\left(22 \pm 2^{\circ} \mathrm{C}, 40 \pm 10 \% \mathrm{RH}\right)$, and subsequently placed in an oven at $70^{\circ} \mathrm{C}$ for eight hours to complete the curing process. The achieved fiber content was 62\% determined by burn-off experiments as described in ASTM D 3171-99 [41]. Rectangular specimens with the dimensions of $250 \times 25 \times 2.3 \mathrm{~mm}^{3}$ (length $\times$ width $\times$ thickness), according to ASTM D3039 [42], were cut from the laminates with a water-jet and two aluminum tabs with dimensions of $45 \times 25 \times 4 \mathrm{~mm}^{3}$ were glued to each specimen end using a low viscosity cyanoacrylate glue for gripping. All tab edges were grinded by machine grinder before use to avoid stress concentrations in the gripping area.

\subsection{Experimental set-up and instrumentation}

All fatigue experiments were carried out according to ASTM D7791-12 [43] on an MTS Landmark servo-hydraulic loading machine, calibrated to a load capacity of $25 \mathrm{kN}$ with $\pm 0.5 \%$ of applied force accuracy. The cyclic loading was performed in the stress range of $58 \mathrm{MPa}-78 \mathrm{MPa}$ to achieve failure at between $10^{3}$ and $10^{7}$ fatigue cycles. Four specimens were examined at each of the five selected stress levels. Details of the experimental results are presented in Table 1. A loading rate of $30.5 \mathrm{kN} / \mathrm{s}$ was used for all tests. Different frequencies were selected to keep the loading rate constant for all stress levels. The experimental matrix in detail is presented in Table 1. All experiments were performed in an environmental chamber regulated to a constant temperature of $20^{\circ} \mathrm{C}$. Two fans were used to circulate the air inside the chamber and cool the specimens.

Different instrumentation was used to monitor the mechanical, thermal, and optical changes in the material during and after the fatigue process. During the fatigue experiments, the machine's displacement, load, and number of cycles were recorded. The variation of the longitudinal strains was measured by a high-resolution video-extensometer (a Point Grey Grasshopper3 camera with a resolution of $1936 \times 1216$ Mpixels and a Fujinon HF35SA-1 35mm F/1.4 lens) with a frequency of acquisition of 160 fps. Depending on the stress level, around 20 (between 17 and 23) load and displacement measurements were recorded in each fatigue cycle. The video-extensometer measured the relative position of two lines marked on the specimen surface and calculated the corresponding strain values. An LED white light with negligible heat emission was projected onto the sample surface to enhance measurement accuracy. To record the evolution of the specimen surface 
temperature during the fatigue experiments, an infrared (IR) thermal camera with an accuracy of $0.1{ }^{\circ} \mathrm{C}$ and optical resolution of $160 \times 120$ pixels was also employed. The fabricated material was semitransparent and the formation and accumulation of the fatigue damage decreased specimen translucency $[6,10]$. Therefore, to detect the damage development in the specimens at a macroscale level, photographs were taken at regular intervals (depending on the life expectancy) with a digital camera with maximum aperture $\mathrm{f} / 2.8$ and focal length range of $24-70 \mathrm{~mm}$ zoom range during loading. A bright white light source was positioned behind the specimens to assist this procedure.

\section{Experimental results and discussion}

\subsection{Fatigue life}

The fatigue data are presented in Fig. 1, where the maximum stress level, $\sigma_{\max }$, is plotted versus the number of cycles to failure, $N_{\mathrm{f}}$. The fatigue behavior was modeled by a single power law equation as follows [44]:

$\sigma_{\max }=\sigma_{0} N^{-\frac{1}{k}}$

in which $\sigma_{0}$, the y-intercept, and $1 / k$, the slope of the S-N curve, are model parameters that were derived by linear regression analysis of the experimental data, and their estimated values are given in Table 2 .

\subsection{Specimen translucency and self-generated temperature}

Figures $2 \mathrm{a}$ and $2 \mathrm{~b}$ show the evolution of specimen translucency at different percentages of specimen fatigue life for high and low cyclic loads. During the cyclic loading, the formation of any form of cracks in the matrix and fiber matrix debonding, when the crack surface was perpendicular to the beam of light, caused light scattering and changed the specimen translucency [10]. Therefore, darker regions in the photos correspond to decreased light transmittance due to greater damage formation. As shown for both cases, damage gradually appeared along the fibers as the number of cycles increased, at around $45^{\circ}$ with respect to the specimen longitudinal axis, which was thus attributed mainly to interface debonding. In addition, at higher stress levels, a necking of the concentrated damage zone was formed as can be seen in Fig. 2a (the initial specimen dimensions are indicated by red dashed lines) and gradually became greater. 
The magnitude and distribution of the self-generated temperature across the surface of specimens at two stress levels of $\sigma_{\max }=78 \mathrm{MPa}$ (high stress level) and $\sigma_{\max }=64$ (low stress level) are shown in Figs. $3 \mathrm{a}$ and $3 \mathrm{~b}$, respectively. At high stress levels, during the early stages of the fatigue life, a uniform distribution of surface temperature was observed and as the number of cycles increased, the selfgenerated temperature increased while its uniformity across the specimen surface was maintained (in the represented temperature scale of $10-30^{\circ} \mathrm{C}$ ). During the final cycles of the fatigue experiment, an oval-shaped hotspot started to appear (see Fig. 3a, after 99\% of fatigue life) oriented in the direction of the fiber bundles, and fatigue failure occurred at the location of the hotspot. These temperature increases were attributed to internal friction in the damaged zones. Lower temperatures were measured at low stress levels, also uniformly distributed across the specimen surface during loading, until only a few cycles before failure, when a hot spot formed.

Figures $4 \mathrm{a}$ and $4 \mathrm{~b}$ show the evolution of the maximum and average surface temperatures of specimens at each stress level. At low and intermediate stress levels, the self-generated temperature initially increased and then, after a certain number of cycles, stabilized until the last cycles of the fatigue experiment. At the highest stress level, the self-generated temperature increased gradually as the number of cycles increased, since the lifetime of this specimen is too short and equilibrium of selfgenerated and dissipated heat is not reached. Finally, fatigue failure occurred with a sudden increase of temperature. The highest maximum temperature was less than $30^{\circ} \mathrm{C}$, remained clearly below $T_{g, \text { onset }}$ $\left(78^{\circ} \mathrm{C}\right)$ measured by DMA [10], and the material thus remained in the glassy state.

\subsection{Stress-strain loops and fatigue stiffness}

The evolution of the typical hysteresis loops during fatigue life at high, $\sigma_{\max }=78 \mathrm{MPa}$, and low, $\sigma_{\max }=$ $58 \mathrm{MPa}$, stress levels is shown in Fig. 5. The number of loading cycles at which the first and the last hysteresis loops were recorded are indicated for both stress levels. To identify the amount of cyclic creep at different stress levels, the variation of the average strain (average of maximum and minimum strains in one cycle) versus the normalized number of cycles is shown in Fig. 6 for selected specimens at each stress level. Cyclic creep was mainly due to the increased viscoelastic deformation of the polymeric matrix [26]. Another reason for the occurrence of cyclic creep is the permanent strain due to fatigue damage accumulation. All curves showed a rapid increase with decreasing rate at the early stage of loading, followed by a steady state evolution prior to an increase before failure. As expected, the specimens loaded at higher stress levels exhibited greater cyclic creep. 
Figure 7 shows part of a specimen at the different percentages of fatigue life at the high stress level. In Fig. 7a, the dashed line indicates the initial position of a selected fiber bundle in the composite laminate first layer before starting the experiment; however, due to the symmetry of the specimen, the realignment angle would be symmetrical with respect to the specimen longitudinal axis, and therefore fiber bundles in all other layers would be realigned at same angles. In addition, the fiber realignment was not uniform along the specimen but was more evident at the concentrated damage zones, as a result of the cyclic creep; the new fiber orientation indicated with solid lines in Figs. 7b-d gradually changed with the number of cycles up to specimen failure.

The average difference of the realignment angle, $\Delta \theta_{a v}$, was obtained from the measurements of the longitudinal and traverse strains. Figure 8 shows schematically the realignment of the fiber angle in a specimen as a result of longitudinal $(\Delta a)$ and traverse $(\Delta b)$ deformations. The average difference of the realignment angle was calculated as:

$\Delta \theta_{a v}=\arctan \left(\frac{1+\varepsilon_{x}}{1-\varepsilon_{y}}\right)-45$

where $\varepsilon_{\mathrm{x}}, \varepsilon_{\mathrm{y}}$ are the longitudinal and traverse strains, respectively as measured. The evolution of the calculated average realignment angle versus the normalized number of cycles is shown in Fig. 9 at different stress levels and was inversely proportional to the evolution of the longitudinal cyclic creep (Fig. 6).

The fatigue stiffness, $E_{N}$, with respect to the stiffness of the first cycle, $E_{l}$, is plotted against the normalized fatigue life for different stress levels in Fig. 10. The evolution of fatigue stiffness was a function of the applied stress level as a result of two opposing mechanisms. On the one hand, as the number of cycles increased, the damage accumulated in the volume of the material and reduced the fatigue stiffness (Fig. 2). On the other hand, the material was stiffened mainly as a result of cyclic creep, which caused the fiber realignment toward the loading direction (Fig. 9). A similar enhancement of fatigue stiffness was also observed in the same specimens when they were subjected to a creep-fatigue loading pattern [7]. Another reason for the increasing fatigue stiffness was attributed to the increasing internal stress of the polymeric matrix, again due to cyclic creep. An enhancement of fatigue stiffness due to increasing internal stress was also observed in an epoxy adhesive during cyclic loading at high stress levels [34]. At the low stress level, the stiffness evolution gradually decreased during the first $15 \%$ of specimen fatigue life followed by a steady state decrease up to specimen failure. This observation is in agreement with the behavior exhibited by most common (FRP) composite systems in the literature, e.g., [10,45,46,47]. However, at high stress levels, the fatigue stiffness increased at the beginning of the experiments due to the fiber realignment, while gradually, as damage accumulated in the specimen, it reached a constant value for most of the specimen fatigue life, 
before decreasing again prior to failure. At $\sigma_{\max }=64 \mathrm{MPa}$, fatigue stiffness was almost constant up to the $90 \%$ of the specimen lifetime as a result of a balance between the stiffening and degradation during the cyclic loading, and then dropped prior to failure due to the prevailing specimen damage. Similarly, almost constant fatigue stiffness during the fatigue experiments was also observed in $( \pm 45)_{2 \mathrm{~S}}$ angle-ply laminates of carbon/epoxy under $R=0.5$; however, no explanation for this behavior was given [26].

The variation of the hysteresis loop area per cycle versus the normalized number of cycles is shown for selected specimens at each stress level in Fig. 11. The hysteresis loop area represents the energy dissipated per cycle mainly due to the internal friction, which increases as the damage area grows $[6,48]$. On the other hand, the increase of fatigue stiffness reduces the internal friction, and consequently the amount of energy dissipated decreases [7]. At high and intermediate stress levels and at the beginning of the experiments, due to the rapidly increasing cyclic creep and resulting stiffening effect, the hysteresis loop area decreased. Subsequently, the area remained the same, due to the balance between the fatigue damage growth and the stiffening effect of fiber realignment, until close to the end of the fatigue life, when the damage became highly concentrated and the amount of internal friction became greater, resulting to a hysteresis loop area increase. At the low stress level, since the damage was not as severe and concentrated, and the mean stress level was low and did not lead to any significant stiffening effect, the hysteresis loop area remained almost constant during the entire lifetime.

\section{Effect of stress ratio}

The S-N curve obtained from the fatigue experiments under the stress ratio of 0.5 together with the $95 \%$ confidence bounds are compared to those derived from experiments under $R=0.1$ [10] in Fig. 1. The fatigue life of specimens under $R=0.5$ was longer than that of specimens under $R=0.1$ at the same $\sigma_{\max }$. The estimated values of the model parameters are given in Table 2; as shown, the slope of the S$\mathrm{N}$ curve was steeper under the stress ratio of 0.1 . It can also be seen that the fatigue data under $R=0.5$

exhibited greater scatter, with a variance of twice the normal distribution, $\hat{\sigma}^{2}$, calculated according to [49] and also tabulated in Table 2. In laminated composites, preexisting defects can vary from specimen to specimen, and fatigue damage initiates at these locations. At low stress amplitudes $(R=0.5)$, damage formation and accumulation were highly dependent on the differences in preexisting defects, which led to lower repeatability in the fatigue results [50]. In addition, when the stress ratio changed from 0.1 to 0.5 , the creep effect became more prevalent, causing more scattered results. The fracture surfaces were examined by using a digital handheld Dino-Lite microscope, AD7013MZT, 
with a magnification of $20 \mathrm{x}$ and resolution of $2592 \times 1944$ pixels. Observation of the photos taken for all specimens shown that specimens loaded at both stress ratios exhibited similar fracture, following the patterns described in details in [10]. At high stress levels, the failure was characterized by extensive fiber pull-out, while at lower cyclic stresses, a mixed-mode failure with fiber pull-out and fiber breakage was observed.

A comparison of the specimen translucency in Fig. 2 with that in [10] indicated that under the stress ratio of 0.5 , more uniform damage distribution was formed along the specimens than in those under the stress ratio of 0.1 . Under the higher stress ratio, when damage initiated, it propagated at a lower rate due to the smaller stress amplitude, which led to more uniform damage distribution. The selfgenerated surface temperature measurements shown in Fig. 3 support this. The temperature across the specimen surface remained even throughout the fatigue experiment, and hot spots were present only during the last $10 \%$ of fatigue life, in contrast to specimens loaded continuously under $R=0.1$ [10] where hot spots could be observed from the early stages of the fatigue experiment [10]. At constant $\sigma_{\max }$, a greater cyclic stress amplitude under $R=0.1$ caused the formation of dominant cracks in the specimen volume creating concentrated damage zones. Nevertheless, under $R=0.5$, the cyclic stress amplitude was much lower, insufficient to create dominant cracks, and therefore fatigue damage was evenly distributed in the specimen volume throughout the fatigue lifetime, which finally led to longer fatigue life.

The evolution of the cyclic creep with the number of cycles under the two studied stress ratios is compared in Fig. 12 for the two stress levels of $\sigma_{\max }=68 \mathrm{MPa}$ and $\sigma_{\max }=58 \mathrm{MPa}$. As the stress ratio increased, at the same $\sigma_{\max }$, the mean stress became greater and the stress amplitude decreased. Therefore, in the experiment with $R=0.5$, the cyclic creep mainly increased because of the viscoelastic strain; however, in the case of $R=0.1$, the cyclic creep was governed by the permanent strain. According to Fig. 12, the cyclic creep initially increased very rapidly in the experiment with $R=0.5$ and almost $70 \%$ of the cyclic creep of the specimens was achieved in the first $20 \%$ of the fatigue life, which was due to the high viscoelastic deformation. However, the strain of specimens loaded under $R=0.1$ increased uniformly as the number of cycles increased because of gradual damage formation and accumulation. Although the initial two stages of the cyclic creep curves under different stress ratios increased at different rates, the strains at failure were comparable. In addition, Fig. 12 shows that the difference between the observed cyclic creep became lesser at the lower stress level. The evolution of the failure strains versus $\sigma_{\max }$ is shown in Fig. 13. A direct relationship between the failure strain and stress level can be observed for both stress ratios. In addition, it can be seen that independent of stress levels, the failure strains were comparable at other stress levels. 
The variation of the average normalized fatigue stiffness $\left(E_{N} / E_{l}\right)$ versus normalized fatigue life is shown in Fig. 14 for the high and low stress levels under both $R=0.1$ and $R=0.5$. At $\sigma_{\max }=68 \mathrm{MPa}$ and $R=0.1$, the normalized fatigue stiffness continuously decreased, initially at a high rate (during the first $10-15 \%$ of the lifetime), and subsequently at a lower, steady state rate up to specimen failure. The stiffness evolution was different for those specimens loaded under $R=0.5$, for which, as explained above, the stiffness increased due to the fiber realignment until sufficient damage developed. The continuous decreasing of the normalized fatigue stiffness under $R=0.1$ was attributed to the greater stress amplitude and lower mean stress than under $R=0.5$, causing more fatigue damage and less stiffening effect. At lower stress levels (e.g., $\sigma_{\max }=58 \mathrm{MPa}$ ), for both stress ratios, fatigue stiffness decreased as the number of cycles increased. At this stress level, the stiffening effect was not sufficiently high to compensate the degradation effect of fatigue damage.

The same comparison of the different stress ratios is shown for the evolution of the hysteresis loop areas in Fig. 15. Under the stress ratio of 0.1, when the number of cycles increased, the hysteresis loop area continuously increased. By increasing the stress ratio from 0.1 to 0.5 , the magnitude of the hysteresis loop area decreased considerably mainly because of the smaller stress amplitude and less damage accumulation, which finally led to lower internal friction. Additionally, under the stress ratio of 0.5 , the mean stress was greater than that at the same stress levels under $R=0.1$, causing more fiber realignment and more specimen stiffening, which again decreased the hysteresis loop area.

\section{Conclusions}

In this work, to determine the stress ratio effect on the fatigue behavior of angle-ply laminated composites, the mechanical, thermal, and optical properties of $( \pm 45)_{2 \mathrm{~s}}$ glass/epoxy composite under two stress ratios of 0.5 and 0.1 were compared at different stress levels. The following conclusions can be drawn:

- By increasing the stress ratio from 0.1 to 0.5 , the fatigue life was enhanced at constant $\sigma_{\max }$, and the slope of the S-N curve decreased with more scattered fatigue results.

- The different stress ratios led to different damage distributions, as shown by the specimen translucency during the fatigue loading. In the experiments conducted under the stress ratio of 0.5 , damage was uniformly distributed along the specimens, while under the stress ratio of 0.1 , it was severe and localized, which caused higher selfgenerated temperatures, and failure of these specimens at shorter lifetimes. 
- The cyclic creep increased due to the viscoelastic deformation and the permanent strain accumulation in a ternary form. Under $R=0.5$, the cyclic creep mainly increased because of the viscoelastic deformation in the primary stage; however, under $R=0.1$, the cyclic creep principally grew as a result of the gradual increase of permanent strain in the steady state stage. Nevertheless, in both cases, the failure strains were comparable.

- The fatigue stiffness evolution during fatigue life was influenced by two opposing mechanisms; fatigue damage that degraded stiffness and a stiffening effect caused mainly by the fiber realignment due to cyclic creep. Under $R=0.5$ and at high stress levels, fiber realignment and the corresponding stiffening effect were significant, which enhanced the fatigue stiffness at the beginning of the fatigue experiments. However, the two mechanisms subsequently became balanced as the number of cycles increased, and finally, the predominant damage growth decreased the fatigue stiffness. At the low stress level, the stiffening effect was not pronounced and therefore the fatigue stiffness monotonically decreased. When the stress ratio was decreased from 0.5 to 0.1 , more severe damage zones and less stiffening effect caused the continuous decrease of fatigue stiffness at all stress levels.

- The variation of the hysteresis loop area during fatigue life was influenced by the same two opposing mechanisms; fatigue damage growth, which increased the internal friction, and the stiffening effect, which decreased the internal friction. Under $R=0.5$ and at high and intermediate stress levels, after a primary stage where the dominant stiffening effect decreased the hysteresis loop area, the damage growth and specimen stiffening became balanced for the greater part of the specimen fatigue life, which resulted in a constant hysteresis loop area. At the end of fatigue life, when the damage growth effect dominated, the hysteresis loop area slightly increased. However, under the stress ratio of 0.1 , the hysteresis loop area continuously increased at all examined stress levels.

\section{Acknowledgments}

The authors wish to acknowledge the support and funding of this research by the Swiss National Science Foundation (Grant No. 200021_156647/1). 


\section{References}

[1] Philippidis, T. P., \& Vassilopoulos, A. P. (2004). Life prediction methodology for GFRP laminates under spectrum loading. Composites Part A: applied science and manufacturing, 35(6), 657-666.

[2] Plumtree, A., Melo, M., \& Dahl, J. (2010). Damage evolution in a $[ \pm 45]_{2 S}$ CFRP laminate under block loading conditions. International Journal of Fatigue, 32(1), 139-145.

[3] Shahverdi, M., Vassilopoulos, A. P., \& Keller, T. (2012). Experimental investigation of R-ratio effects on fatigue crack growth of adhesively-bonded pultruded GFRP DCB joints under CA loading. Composites Part A: Applied Science and Manufacturing, 43(10), 1689-1697.

[4] Mandell, J. F., Samborsky, D. D., Miller, D. A., Agastra, P., \& Sears, A. T. (2016). Analysis of SNL/MSU/DOE Fatigue Database Trends for Wind Turbine Blade Materials 2010-2015(No. SAND2016-1441). Sandia National Lab.(SNL-NM), Albuquerque, NM (United States).

[5] Pelaseyed, S. S., Mashayekhi, F., \& Movahedi-Rad, A. (2015). Investigation of the shaft failure connected to extruder. Journal of Failure Analysis and Prevention, 15(6), 775-781.

[6] Movahedi-Rad, A. V., Keller, T., \& Vassilopoulos, A. P. (2018). Interrupted tension-tension fatigue behavior of angle-ply GFRP composite laminates. International Journal of Fatigue, 113, 377388.

[7] Movahedi-Rad, A. V., Keller, T., \& Vassilopoulos, A. P. (2019). Creep effects on tension-tension fatigue behavior of angle-ply GFRP composite laminates. International Journal of Fatigue. 123, 144156.

[8] Vassilopoulos, A. P., \& Keller, T. (2011). Fatigue of fiber-reinforced composites. Springer Science \& Business Media.

[9] Reifsnider, K. L., \& Talug, A. (1980). Analysis of fatigue damage in composite laminates. International Journal of Fatigue, 2(1), 3-11.

[10] Movahedi-Rad, A. V., Keller, T., \& Vassilopoulos, A. P. (2018). Fatigue damage in angle-ply GFRP laminates under tension-tension fatigue. International Journal of Fatigue, 109, 60-69.

[11] Salkind, M. J. (1972). Fatigue of composites. In Composite Materials: Testing and Design (Second Conference). ASTM International.

[12] Rotem, A. (1991). The fatigue behavior of composite laminates under various mean stresses. Composite structures, 17(2), 113-126.

[13] Kawai, M. (2004). A phenomenological model for off-axis fatigue behavior of unidirectional polymer matrix composites under different stress ratios. Composites Part A: applied science and manufacturing, 35(7-8), 955-963. 
[14] Kawai, M., \& Kato, K. (2006). Effects of R-ratio on the off-axis fatigue behavior of unidirectional hybrid GFRP/Al laminates at room temperature. International journal of fatigue, 28(10), 1226-1238.

[15] Brunbauer, J., \& Pinter, G. (2015). Effects of mean stress and fibre volume content on the fatigueinduced damage mechanisms in CFRP. International Journal of Fatigue, 75, 28-38.

[16] Sun, C. T., \& Chan, W. S. (1979). Frequency effect on the fatigue life of a laminated composite. In Composite materials: testing and design (fifth conference). ASTM International.

[17] Sun, C. T., \& Chim, E. S. (1981). Fatigue retardation due to creep in a fibrous composite. In Fatigue of Fibrous Composite Materials. ASTM International.

[18] Perreux, D., \& Joseph, E. (1997). The effect of frequency on the fatigue performance of filamentwound pipes under biaxial loading: experimental results and damage model. Composites science and technology, 57(3), 353-364.

[19] Barron, V., Buggy, M., \& McKenna, N. H. (2001). Frequency effects on the fatigue behaviour on carbon fibre reinforced polymer laminates. Journal of materials science, 36(7), 1755-1761.

[20] Philippidis, T. P., \& Vassilopoulos, A. P. (2003). Fatigue strength of composites under variable plane stress. In Fatigue in Composites (pp. 504-525).

[21] El Kadi, H., \& Ellyin, F. (1994). Effect of stress ratio on the fatigue of unidirectional glass fibre/epoxy composite laminae. Composites, 25(10), 917-924.

[22] Epaarachchi, J. A., \& Clausen, P. D. (2003). An empirical model for fatigue behavior prediction of glass fibre-reinforced plastic composites for various stress ratios and test frequencies. Composites Part A: Applied science and manufacturing, 34(4), 313-326.

[23] Vassilopoulos, A. P., Manshadi, B. D., \& Keller, T. (2010). Influence of the constant life diagram formulation on the fatigue life prediction of composite materials. International journal of fatigue, 32(4), 659-669.

[24] Andersons, J., \& Paramonov, Y. (2011). Applicability of empirical models for evaluation of stress ratio effect on the durability of fiber-reinforced creep rupture-susceptible composites. Journal of materials science, 46(6), 1705-1713.

[25] Huh, Y. H., Lee, J. H., Kim, D. J., \& Lee, Y. S. (2012). Effect of stress ratio on fatigue life of GFRP composites for WT blade. Journal of mechanical science and technology, 26(7), 2117-2120.

[26] Petermann, J., \& Schulte, K. (2002). The effects of creep and fatigue stress ratio on the long-term behaviour of angle-ply CFRP. Composite Structures, 57(1-4), 205-210. 
[27] Miyano, Y., Nakada, M., McMurray, M. K., \& Muki, R. (1997). Prediction of flexural fatigue strength of CRFP composites under arbitrary frequency, stress ratio and temperature. Journal of Composite Materials, 31(6), 619-638.

[28] Guedes, R. M. (2007). Durability of polymer matrix composites: Viscoelastic effect on static and fatigue loading. Composites Science and Technology, 67(11-12), 2574-2583.

[29] Mallick, P. K., \& Zhou, Y. (2004). Effect of mean stress on the stress-controlled fatigue of a short E-glass fiber reinforced polyamide-6, 6. International journal of fatigue, 26(9), 941-946.

[30] Vieille, B., Albouy, W., \& Taleb, L. (2014). About the creep-fatigue interaction on the fatigue behaviour of off-axis woven-ply thermoplastic laminates at temperatures higher than Tg. Composites Part B: Engineering, 58, 478-486.

[31] Sayyidmousavi, A., Bougherara, H., \& Fawaz, Z. (2015). The role of viscoelasticity on the fatigue of angle-ply polymer matrix composites at high and room temperatures-a micromechanical approach. Applied Composite Materials, 22(3), 307-321.

[32] Vinogradov, A. M., \& Schumacher, S. (2001). Cyclic creep of polymers and polymer-matrix composites. Mechanics of composite materials, 37(1), 29-34.

[33] Stelzer, S., Ucsnik, S., \& Pinter, G. (2015). Fatigue behaviour of composite-composite joints reinforced with cold metal transfer welded pins. International Journal of Fatigue, 81, 37-47.

[34] Savvilotidou, M., Keller, T., \& Vassilopoulos, A. P. (2017). Fatigue performance of a cold-curing structural epoxy adhesive subjected to moist environments. International Journal of Fatigue, 103, 405-414.

[35] Raghavan, J., \& Meshii, M. (1997). Creep rupture of polymer composites. Composites Science and Technology, 57(4), 375-388.

[36] Enyama, J., McMurry, M.K., Nakada M., \& Miyano Y. (1993). Effects of stress ratio on flexural fatigue behavior of a satin woven CFRP laminate. In Proc. 3rd Japan SAMPE (Vol. 2, pp. 24182421).

[37] Zhang, Y., Vassilopoulos, A. P., \& Keller, T. (2008). Stiffness degradation and fatigue life prediction of adhesively-bonded joints for fiber-reinforced polymer composites. International Journal of Fatigue, 30(10-11), 1813-1820.

[38] Ma, Y., Zhang, Y., Sugahara, T., Jin, S., Yang, Y., \& Hamada, H. (2016). Off-axis tensile fatigue assessment based on residual strength for the unidirectional 45 carbon fiber-reinforced composite at room temperature. Composites Part A: Applied Science and Manufacturing, 90, 711-723.

[39] Ziemian, C. W., Ziemian, R. D., \& Haile, K. V. (2016). Characterization of stiffness degradation caused by fatigue damage of additive manufactured parts. Materials \& Design, 109, 209-218. 
[40] Fawaz, Z., \& Ellyin, F. (1994). Fatigue failure model for fibre-reinforced materials under general loading conditions. Journal of Composite Materials, 28(15), 1432-1451.

[41] ASTM, D3171-99, (2002). Standard test methods for constituent content of composite materials. ASTM International.

[42] ASTM D3039/D3039M-14, (2014). Standard Test Method for Tensile Properties of Polymer Matrix Composite Materials. ASTM International.

[43] ASTM, D7791-17, (2017). Standard Test Method for Uniaxial Fatigue Properties of Plastics. ASTM International.

[44] Sarfaraz, R., Vassilopoulos, A. P., \& Keller, T. (2012). A hybrid S-N formulation for fatigue life modeling of composite materials and structures. Composites Part A: Applied Science and Manufacturing, 43(3), 445-453.

[45] Reifsnider, K. L., Schulte, K., \& Duke, J. C. (1983). Long-term fatigue behavior of composite materials. In Long-term behavior of composites. ASTM International.

[46] Philippidis, T. P., \& Vassilopoulos, A. P. (1999). Fatigue of composite laminates under off-axis loading. International Journal of fatigue, 21(3), 253-262.

[47] Mivehchi, H., \& Varvani-Farahani, A. (2010). The effect of temperature on fatigue strength and cumulative fatigue damage of FRP composites. Procedia Engineering, 2(1), 2011-2020.

[48] Chandra, R., Singh, S. P., \& Gupta, K. (1999). Damping studies in fiber-reinforced composites-a review. Composite structures, 46(1), 41-51.

[49] ASTM E739-10 (Reapproved 2015). Statistical analysis of linear or linearized stress-life (S-N) and strain-life $(\varepsilon-\mathrm{N})$ fatigue data. Annual Book of ASTM Standards, 3.

[50] Schijve, J. (2001). Fatigue of structures and materials. Springer Science \& Business Media. 
Table 1. Fatigue experimental results

\begin{tabular}{ccccc}
\hline No. & Code & $\begin{array}{c}\sigma_{\max } \\
(\mathrm{MPa})\end{array}$ & Frequency $(\mathrm{Hz})$ & $N_{f}$ \\
\hline 1 & Conf-0.5-78-a & 78.0 & 6.8 & 20505 \\
2 & Conf-0.5-78-b & 78.0 & 6.8 & 5206 \\
3 & Conf-0.5-78-c & 78.0 & 6.8 & 1767 \\
4 & Conf-0.5-78-d & 78.0 & 6.8 & 1471 \\
5 & Conf-0.5-72-a & 72.0 & 7.4 & 60130 \\
6 & Conf-0.5-72-b & 72.0 & 7.4 & 61616 \\
7 & Conf-0.5-72-c & 72.0 & 7.4 & 36435 \\
8 & Conf-0.5-72-d & 72.0 & 7.4 & 6513 \\
9 & Conf-0.5-68-a & 68.0 & 7.8 & 5436 \\
10 & Conf-0.5-68-b & 68.0 & 7.8 & 112681 \\
11 & Conf-0.5-68-c & 68.0 & 7.8 & 119132 \\
12 & Conf-0.5-68-d & 68.0 & 7.8 & 16642 \\
13 & Conf-0.5-64-a & 64.2 & 8.4 & 76431 \\
14 & Conf-0.5-64-b & 64.2 & 8.4 & 228923 \\
15 & Conf-0.5-64-c & 64.2 & 8.4 & 345818 \\
16 & Conf-0.5-64-d & 64.2 & 8.4 & 108719 \\
17 & Conf-0.5-58-a & 58.2 & 9.1 & 2110761 \\
18 & Conf-0.5-58-b & 58.2 & 9.1 & 295972 \\
19 & Conf-0.5-58-c & 58.2 & 9.1 & 645568 \\
20 & Conf-0.5-58-d & 58.2 & 9.1 & 5245384 \\
\hline
\end{tabular}

Table 2. Fatigue parameters for continuous loading patterns under stress ratios of 0.1 and 0.5

\begin{tabular}{llll}
\hline Stress ratio & $\sigma_{0}$ & $1 / k$ & $\hat{\sigma}^{2}$ \\
\hline$R=0.1$ & 106.09 & 0.0622 & 0.025 \\
$R=0.5$ & 122.54 & 0.0540 & 0.137 \\
\hline
\end{tabular}




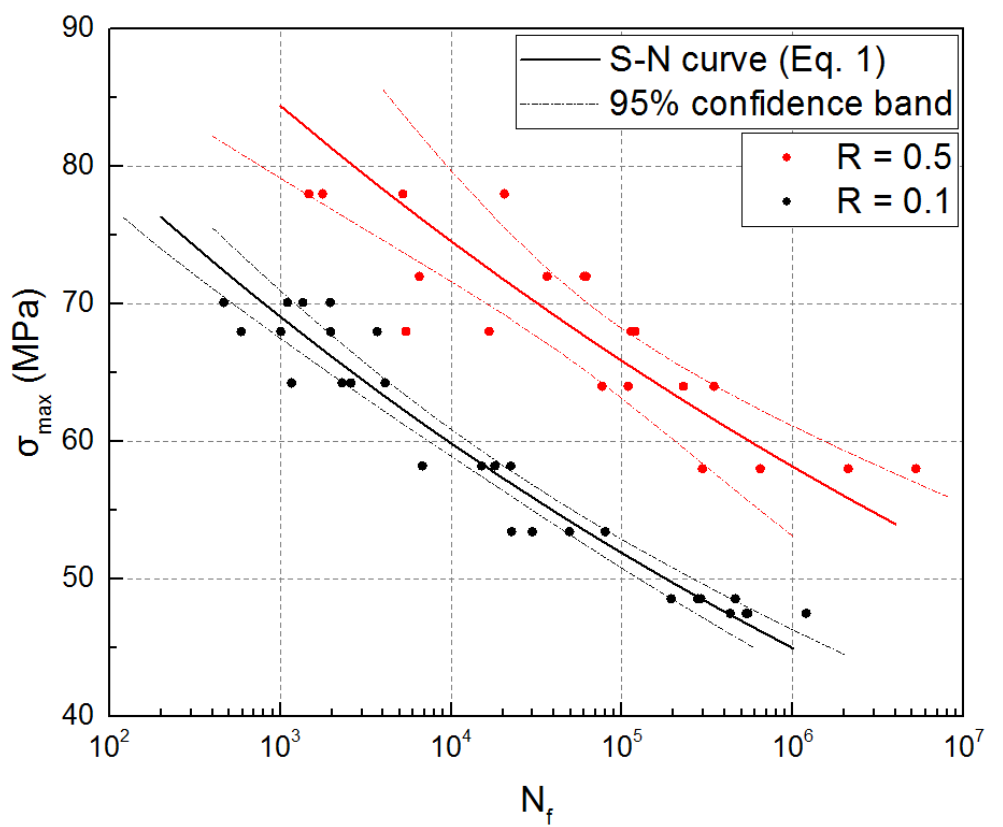

Fig. 1. Comparison of S-N curves under different $R$-ratios. Data points for $R=0.1$ from [10]. 


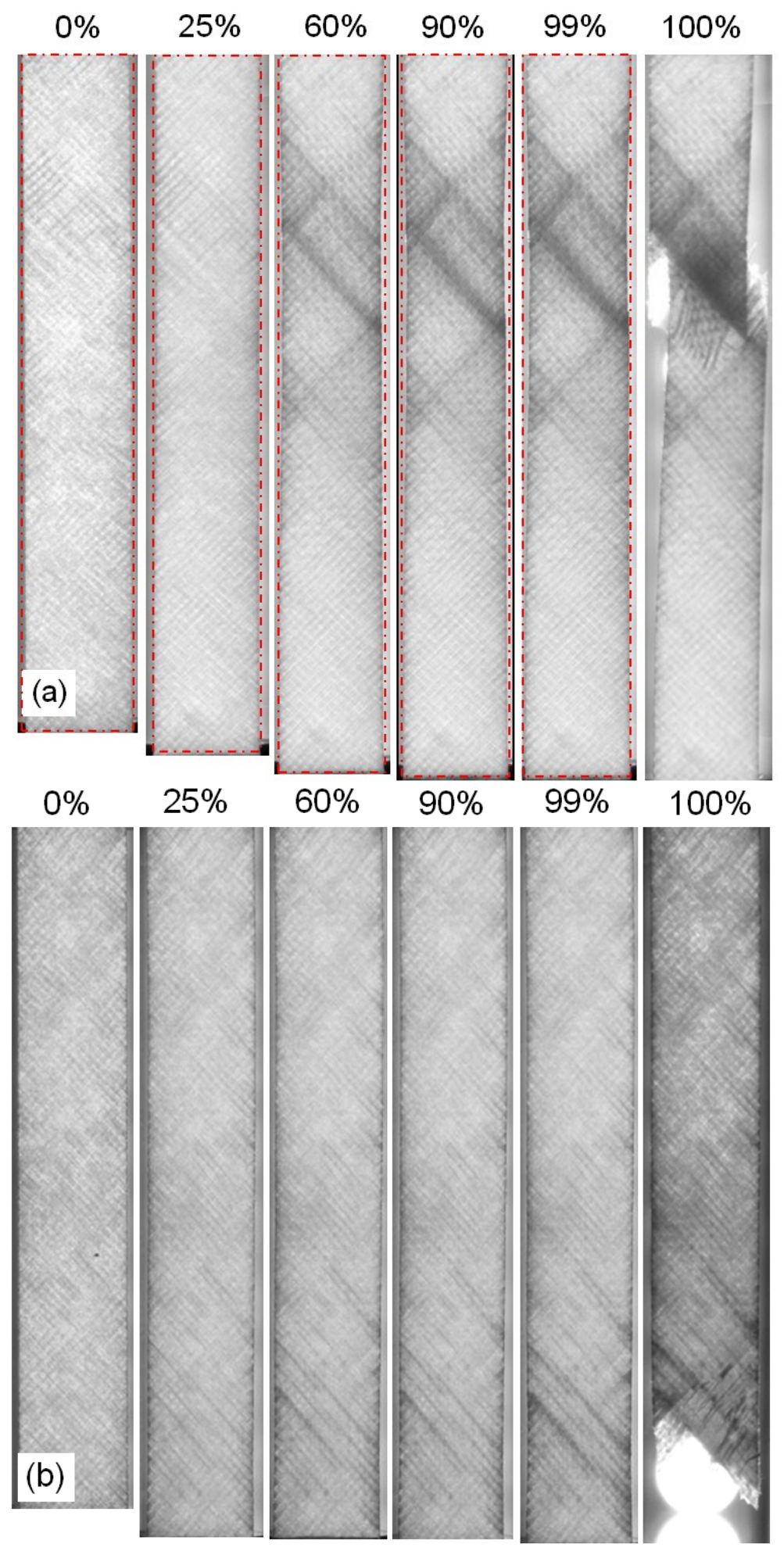

Fig. 2. Light transmittance at different percentages of fatigue life, at (a) $\sigma_{\max }=78 \mathrm{MPa}$ (Conf-0.5-78-b), and (b) $\sigma_{\max }=58 \mathrm{MPa}($ Conf- $0.5-58-\mathrm{d})$. 



Fig. 3. Self-generated temperature at different percentages of fatigue life, at (a) $\sigma_{\max }=78 \mathrm{MPa}$ (Conf-0.5-78-d), and (b) $\sigma_{\max }=64 \mathrm{MPa}$ (Conf-0.5-64-d). 

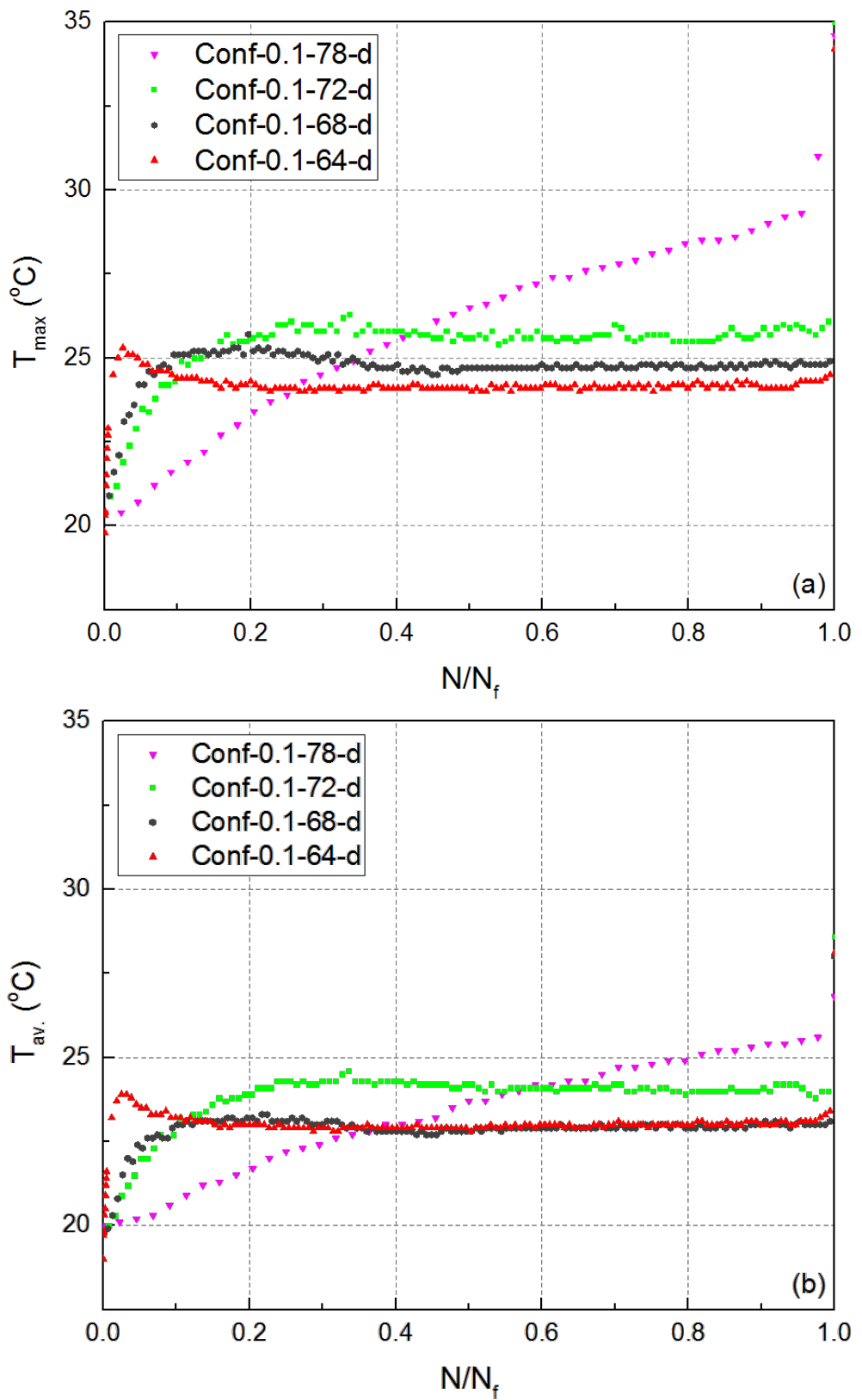

Fig. 4. Variation of (a) maximum, and (b) average self-generated temperature versus normalized number of cycles at different stress levels. 




Fig. 5. Variation of hysteresis loops under cyclic loading at stress levels of $\sigma_{\max }=78 \mathrm{MPa}$ (Conf-0.5-78-a) and $\sigma_{\max }=58 \mathrm{MPa}($ Conf- $0.5-58-\mathrm{a})$.

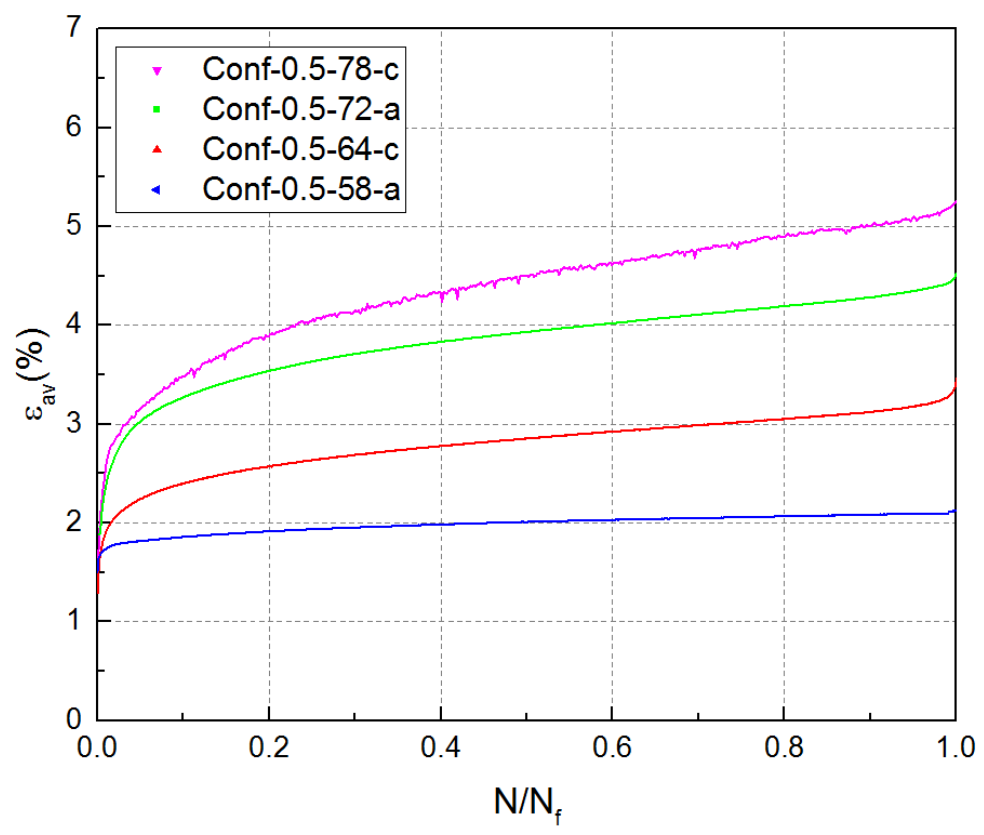

Fig. 6. Cyclic creep versus normalized number of cycles at different stress levels. 

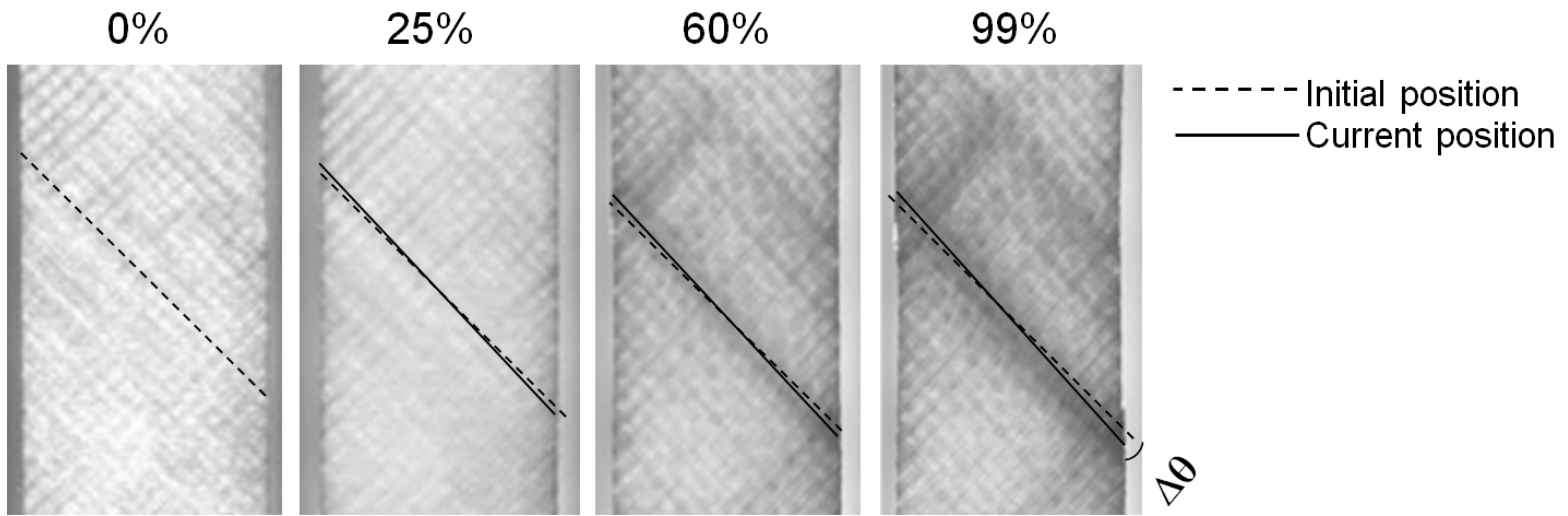

Fig. 7. Realignment of glass fiber bundles at (a) $0 \%$, (b) $25 \%$, (c) $60 \%$, and (d) $99 \%$ of fatigue life (Conf- $0.5-78$ b).

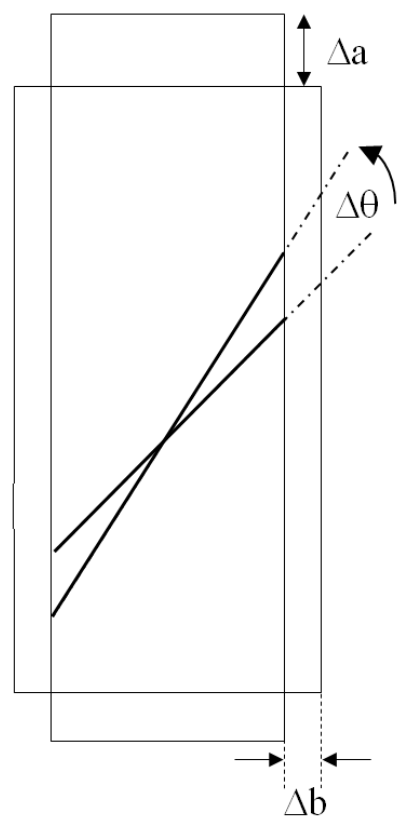

Fig. 8. Schematic representation of realignment angle. 


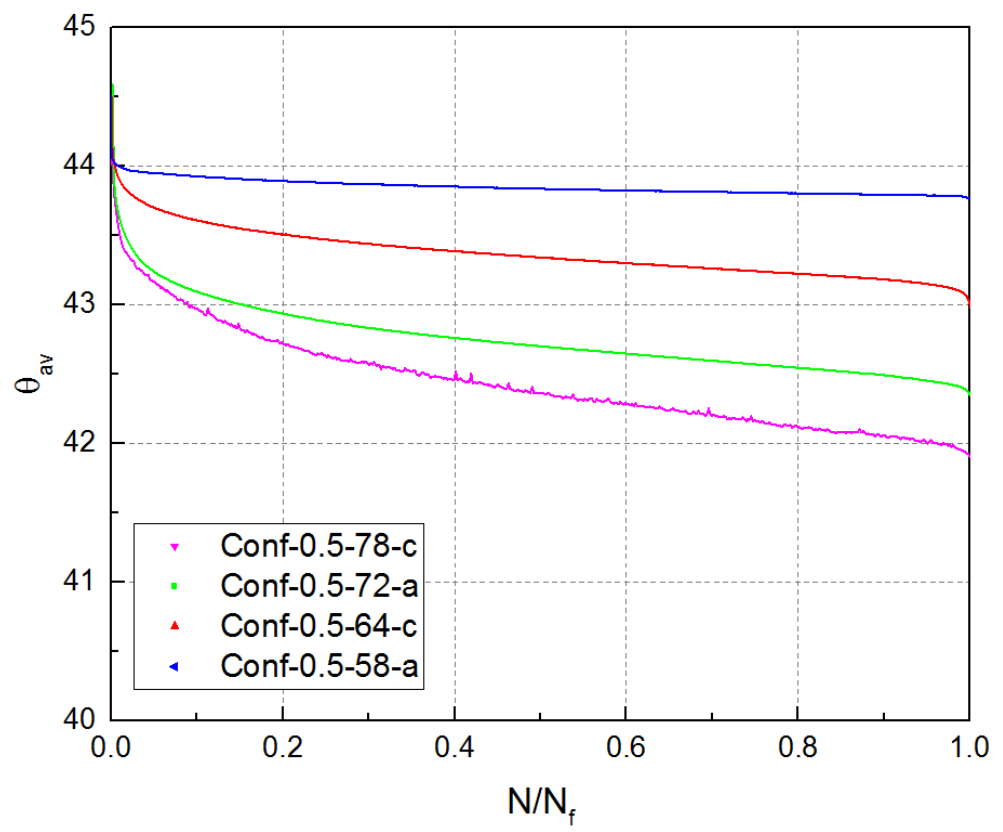

Fig. 9. Average realignment of fibers at different percentages of fatigue life.

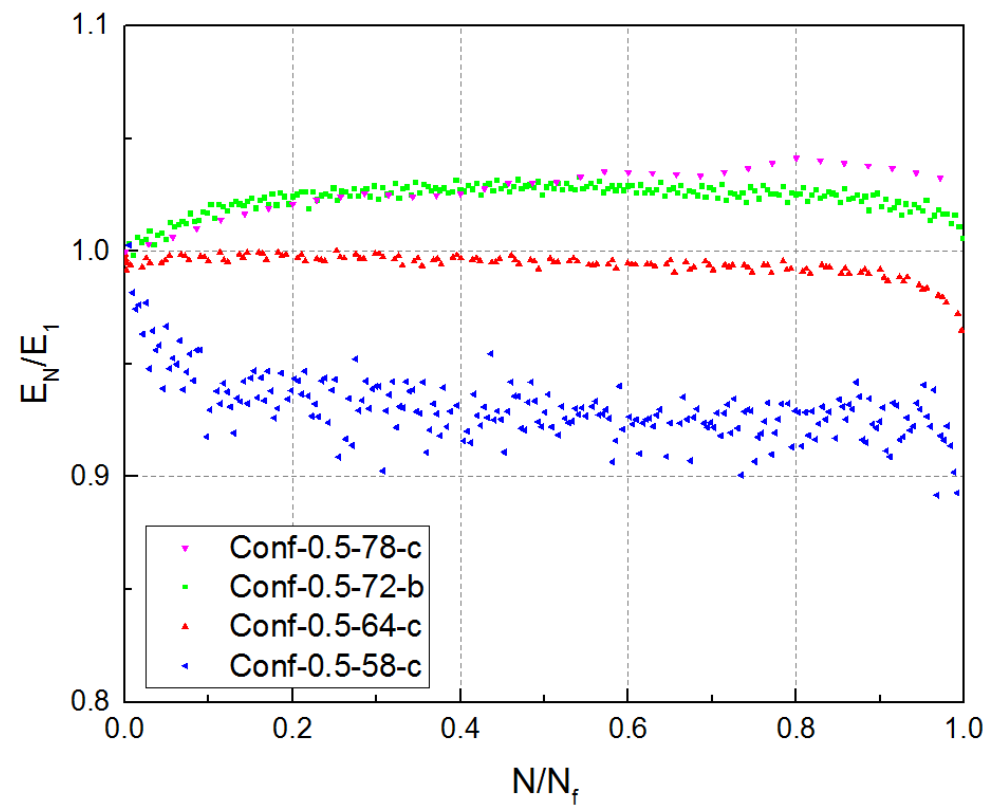

Fig. 10. Variation of normalized fatigue stiffness versus normalized number of cycles at different stress levels. 


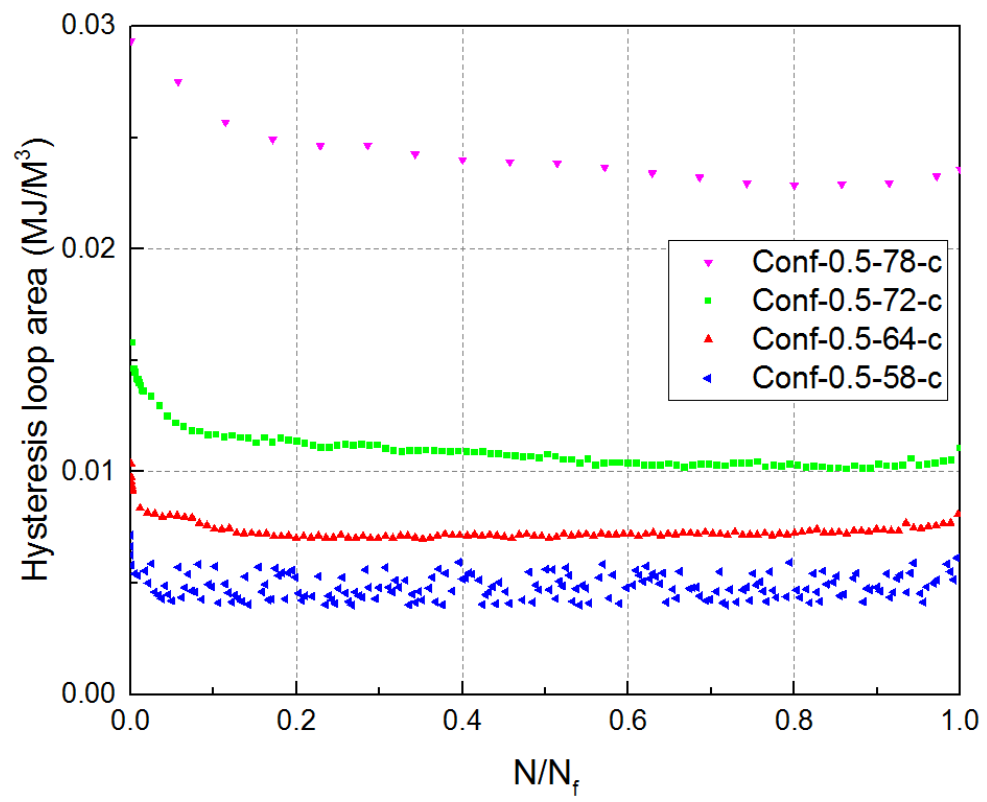

Fig. 11. Hysteresis area per cycle versus normalized number of cycles at different stress levels.

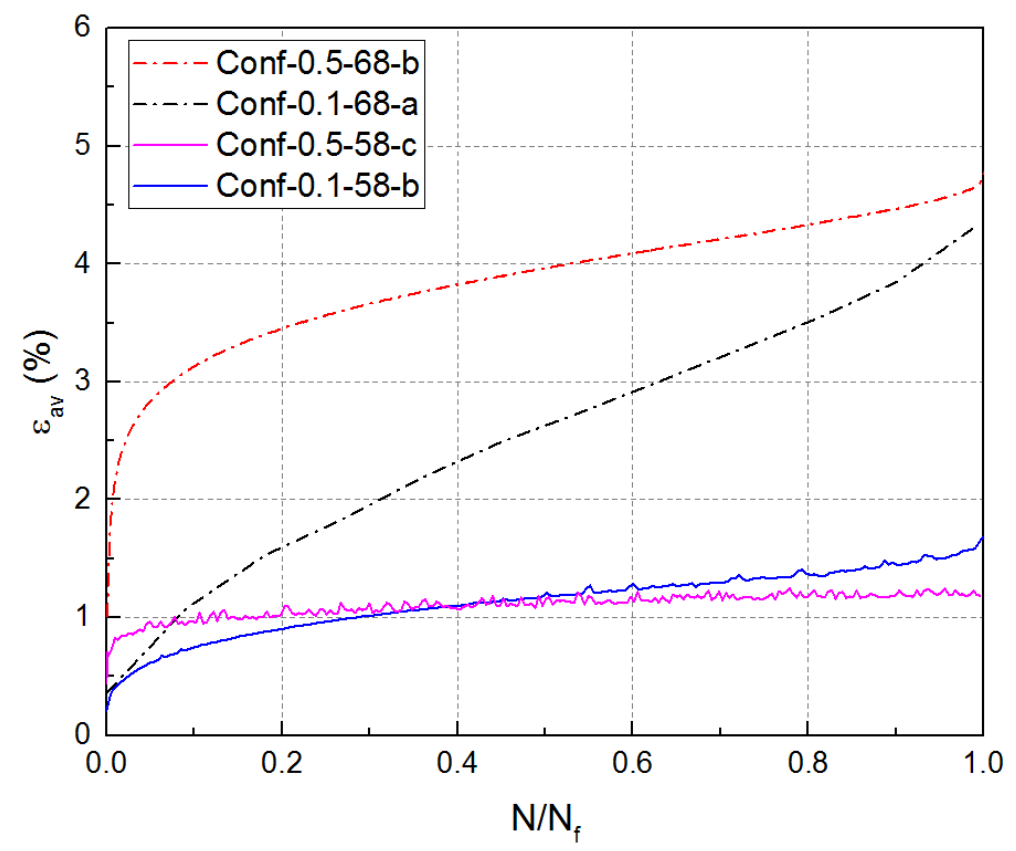

Fig. 12. Cyclic creep versus normalized number of cycles loaded under different stress ratios and at different stress levels. Data points for $R=0.1$ from ref [10]. 


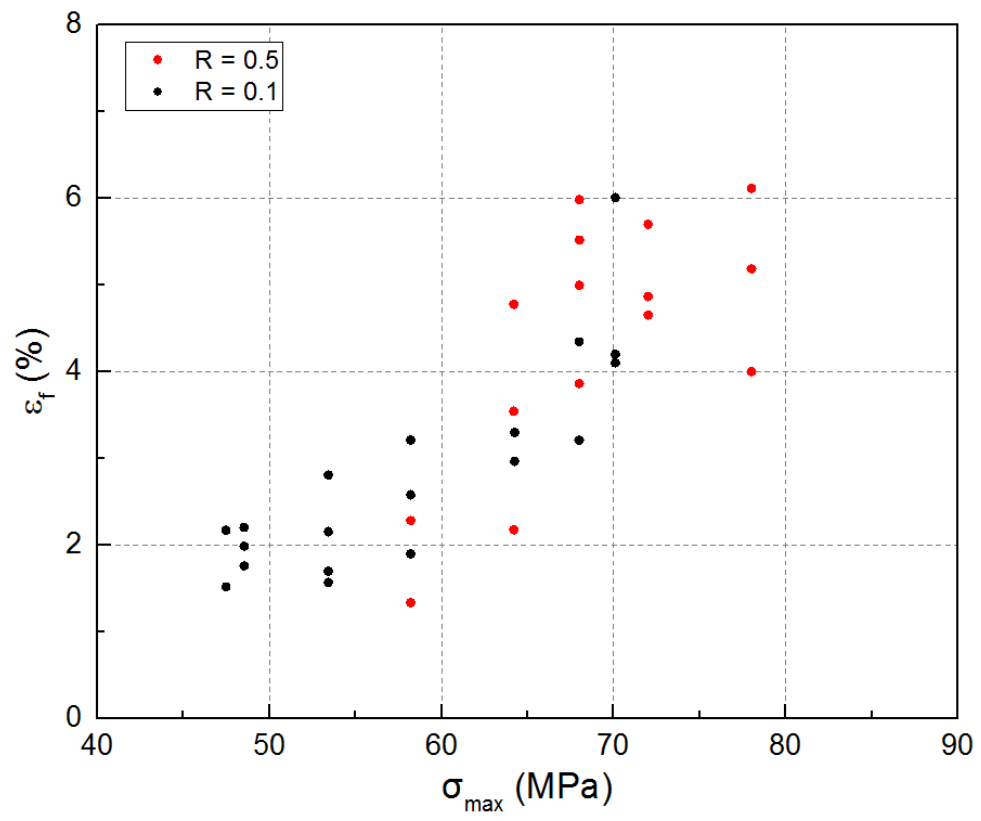

Fig. 13. Failure strain under different stress ratios and at different stress levels. Data points for $R=0.1$ from ref [10].

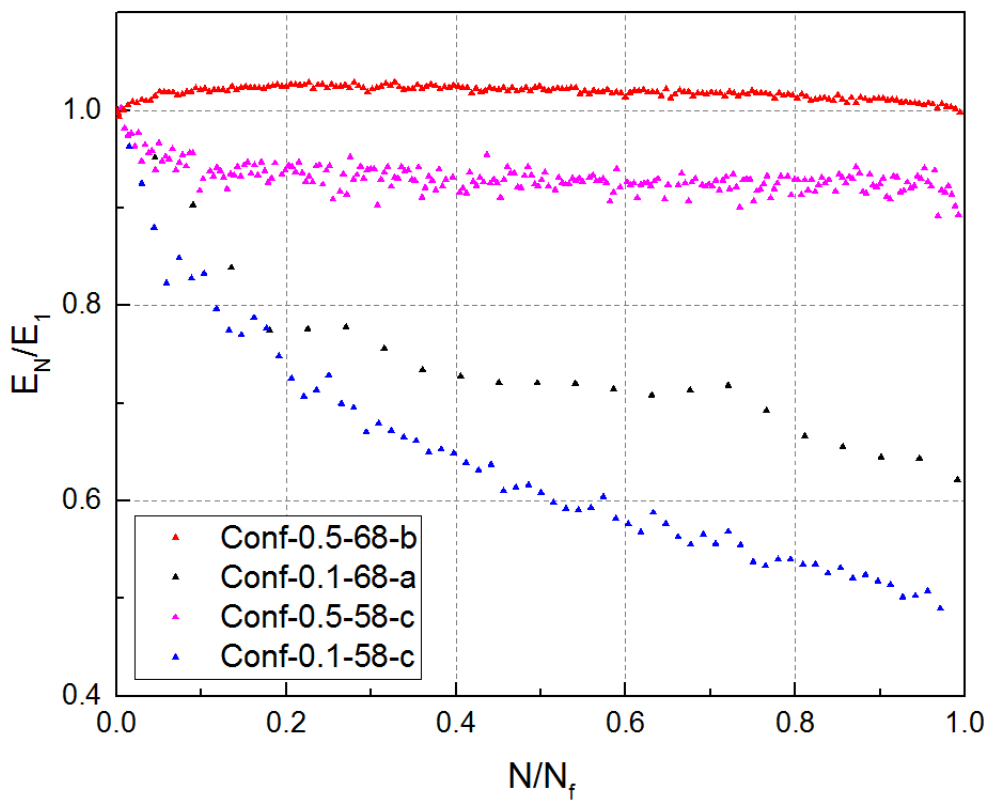


Fig. 14. Normalized fatigue stiffness versus normalized number of cycles loaded under different stress ratios and at different stress levels. Data points for $R=0.1$ from ref [10].

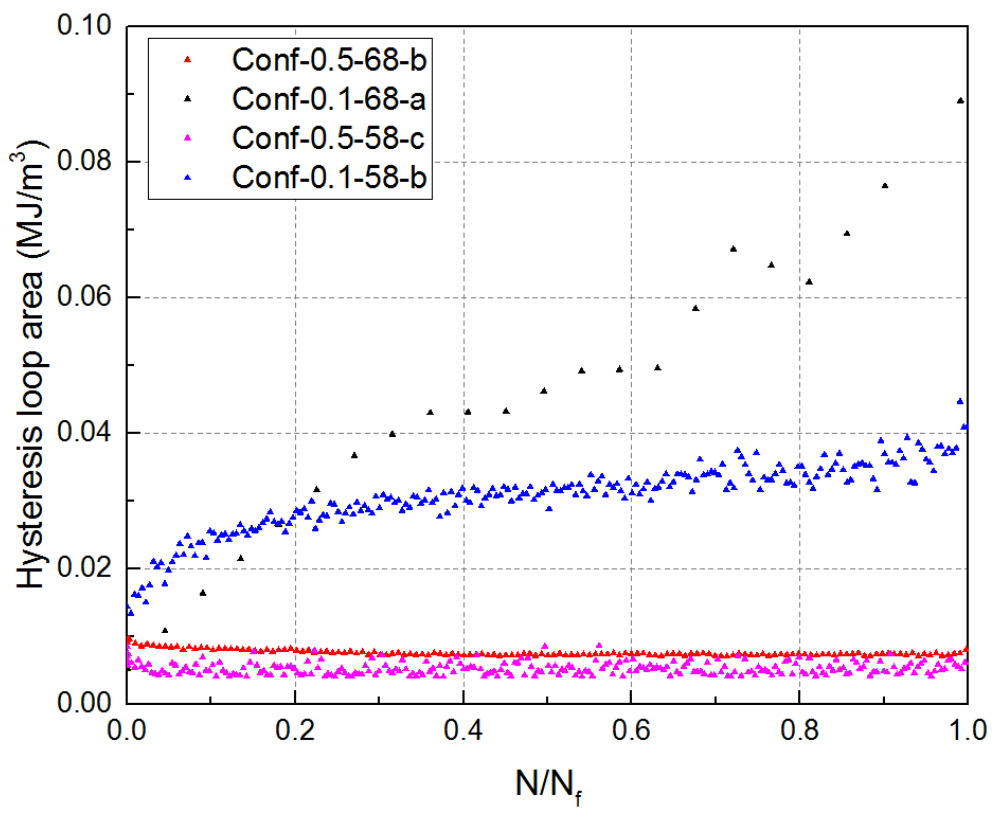

Fig. 15. Hysteresis loop area versus normalized number of cycles loaded under different stress ratios and at different stress levels. Data points for $R=0.1$ from ref [10]. 